\title{
Comportamento verbal: uma análise da abordagem skinneriana e das extensões explicativas de Stemmer, Hayes e Sidman
}

\author{
Ana Leda de Faria Brino \\ Carlos B. A. de Souza \\ Universidade Federal do Pará
}

\begin{abstract}
RESUMO
Para Skinner comportamento verbal é um operante modelado e mantido por conseqüências mediadas por outros. Portanto, poderia ser explicado por meio da descrição de relações funcionais organismoambiente. Entretanto, ao categorizar os operantes verbais Skinner não tratou satisfatoriamente com comportamentos de tipo relacional, provavelmente em função da dificuldade em determinar suas variáveis de controle. Este trabalho procurou analisar a abordagem skinneriana do comportamento verbal e três propostas que buscam ampliá-la (Stemmer, Hayes e Sidman). Stemmer amplia o alcance da explicação skinneriana descrevendo processos de aprendizagem relacional e sintático-gramatical em termos do estabelecimento de discriminações e generalizações, enfatizando o papel de ouvinte como condição necessária à aprendizagem de falante. Hayes e Sidman apresentam, respectivamente, a descrição de um novo tipo de operante e de um novo processo comportamental subjacente à aprendizagem de relações. Ainda que as propostas de Hayes e Sidman estejam alcançando resultados promissores no ensino de repertórios relacionais, não existe um consenso sobre quais mecanismos e processos bio-comportamentais são necessários para a aquisição e o desenvolvimento lingüístico. Sugere-se a necessidade de uma padronização conceitual no que se refere às noções de aprendizagem relacional, classes de estímulos e classes de respostas generalizadas, visando um re-direcionamento das pesquisas sobre comportamento verbal.
\end{abstract}

Palavras-chave: comportamento verbal; equivalência de estímulos; teoria do quadro relacional; eventos ostensivos.

\section{ABSTRACT \\ Verbal Behavior: analysis of the skinnerian perspective and the explaining extensions of Stemmer, Hayes and Sidman}

Skinner defines verbal behavior as an operant shaped and maintained by consequences mediated by others. Therefore, it could be explained through the description of organism-environment functional relations. However, in his categorization of verbal operants Skinner didn't treat satisfactorily with behaviors of relational type, probably due to the difficulty to determine the controlling variables. This work analyzed Skinnerian approach to verbal behavior and three proposals that aim to extend it (Stemmer, Hayes and Sidman). Stemmer extends the scope of Skinnerian explanation describing relational and syntactic-grammatical learning processes in terms of the establishment of discriminations and generalizations, emphasizing the listening role as a necessary condition to the learning of speaking. Hayes and Sidman present, respectively, the description of a new kind of operant and a new behavioral process underlying relational learning. Although Hayes and Sidman's proposals are reaching interesting results in teaching relational repertoires, there is not a consensus about what mechanisms and biobehavioral processes are necessary to the language acquisition and development. This work points to the need of a standardization of concepts related to the notions of relational learning, stimuli classes formation, and generalized response classes, in order to redirect the research on verbal behavior.

Keywords: verbal behavior; stimulus equivalence; relational frame theory; ostensive events. 
A proposta de Skinner (1957) para tratar com o comportamento verbal tem gerado, basicamente, estudos que procuram esclarecer as relações entre os operantes verbais definidos como elementares por ele (ecóico, textual, ditado, cópia, intraverbal, tacto e mando, definidos no item II a seguir). A ausência de um tratamento claro sobre o processo de aquisição desses operantes e sobre a aprendizagem de operantes verbais não-elementares (de segunda ordem ou relacionais), incluindo a aprendizagem sintático-gramatical e suas extensões, deu origem a inúmeras críticas àquela proposta (e.g., Pinker, 1994), e à caracterização da análise do comportamento como uma área incapaz de lidar adequadamente com o fenômeno lingüístico (e.g., Chomsky, 1959; Fodor, 1983). O presente trabalho tratará de analisar 1) a abordagem skinneriana do comportamento verbal e algumas de suas dificuldades, e 2) três propostas - de Natham Stemmer, Steven Hayes e Murray Sidman - que tratam de avançar na compreensão do responder verbal relacional, desde uma perspectiva analítico-comportamental.

Inicialmente o presente trabalho apresenta a definição de comportamento verbal dada por Skinner (1957), em termos da necessidade de uma análise funcional. A seguir apresenta-se a categorização dos operantes verbais elementares e uma primeira avaliação dos seus critérios de categorização. Depois, apresenta-se a definição de responder verbal de segunda ordem de Skinner (1957), avaliada como insuficiente no que concerne à descrição das variáveis (estímulo controlador e consequiências) envolvidas em seu estabelecimento e manutenção. Na seqüência, são apresentadas três propostas analítico-comportamentais (Stemmer, Hayes e Sidman) que têm procurado avançar na discussão sobre o responder verbal relacional. Por fim, discute-se a necessidade de uma padronização conceitual na área, no que se refere às noções de aprendizagem relacional, classes de estímulos e classes de respostas generalizadas, como um passo fundamental para uma reorientação das pesquisas sobre comportamento verbal.

\section{I) A definição de comportamento verbal de Skinner}

Skinner (1957) definiu inicialmente o comportamento verbal como comportamento estabelecido e mantido por consequiências mediadas por outras pessoas. Ao definir desta maneira o comportamento verbal, Skinner (1957) se mantém coerente com seu trabalho anterior, argumentando que a determinação de grande parte do repertório comportamental dos organismos, humanos ou não-humanos, é função de variáveis ambientais e pode, como tal, ser descrita em ter- mos de contingências de reforçamento comportamento-ambiente. A contingência seria, dessa forma, responsável pelo estabelecimento e manutenção do comportamento operante, por meio das consequiências que o comportamento produz no ambiente. Uma vez que um evento ambiental está presente no momento em que determinado comportamento gera a conseqüência, este evento, em situações similares futuras, passa a ter uma maior probabilidade de evocar tal operante: estabelece-se assim o controle de estímulo.

Portanto, o comportamento verbal, para Skinner (1957), deve ser definido e analisado (como qualquer outro comportamento) em termos das contingências de reforçamento, mas levando em conta as diferenças fundamentais de que 1) as conseqüências a este tipo de comportamento seriam fornecidas por um mediador (ouvinte) e 2) que este mediador seja alguém que faz parte da comunidade verbal na qual o falante está inserido, sendo, deste modo, capaz de responder adequadamente aos diferentes operantes verbais emitidos pelo falante.

Desta forma, o operante verbal poderia ser compreendido por meio de uma análise funcional das contingências às quais o organismo é exposto dentro de sua comunidade verbal - o controle de estímulos sobre o comportamento poderia aí ser encontrado, bem como as conseqüências que o estabelecem e mantém.

\section{II) A categorização do comportamento verbal por Skinner: operantes verbais elementares ${ }^{1}$}

Embora o comportamento verbal tenha sido definido com ênfase na importância da análise funcional para a descrição de suas variáveis de controle e verificação da unidade verbal, ao classificar as categorias básicas de comportamento verbal, Skinner (1957) baseou-se principalmente na identificação da natureza do controle dos estímulos antecedentes e sua relação com as características dos produtos das respostas do organismo que o referido estímulo controla (no que concerne à presença ou ausência de similaridade for$\mathrm{mal}^{2}$ (SF) ou correspondência ponto-a-ponto ${ }^{3}$ (CPP) com o estímulo controlador).

As categorias de comportamento verbal elementares, controladas por estímulos verbais antecedentes definidas por Skinner (1957), foram as seguintes: 1) o comportamento ecóico, definido como comportamento verbal controlado por estímulo auditivo antecedente produto da resposta verbal de outrem, cujo produto da resposta, também audível, apresentaria SF e CPP com seu antecedente; 2) o comportamento de copiar, cujo antecedente seria um texto escrito ou 
impresso, e cujo produto da resposta, também um texto escrito ou impresso, apresentaria SF e CPP com seu antecedente; 3 ) o comportamento de (tomar) ditado, cujo antecedente seria um estímulo auditivo produto da resposta vocal de outrem, o qual controlaria uma resposta escrita que, desta forma, apresentaria CPP com seu antecedente, mas não SF; 4) o comportamento textual, cujo antecedente seria um texto impresso, que evocaria uma resposta vocal, havendo, deste modo, CPP entre antecedente e produto de resposta, mas não SF; e 5) o comportamento intraverbal, no qual um estímulo verbal antecedente controla uma resposta verbal, mas sem que exista CPP nem SF entre $\mathrm{o}$ antecedente e a resposta ${ }^{4}$. O reforço generalizado seria a consequiência social responsável pelo estabelecimento e manutenção de todos estes tipos de operantes verbais.

Além dos operantes verbais primários controlados por estímulos verbais antecedentes, outras duas classes de operantes verbais primários foram definidas: o mando e o tacto. $\mathrm{O}$ tacto, também estabelecido e mantido via reforçamento generalizado, apresentaria como estímulo controlador antecedente um estímulo não-verbal, ou seja, um objeto ou evento ou aspectos de objetos e eventos do ambiente. Um exemplo seria, dada a presença da mãe, uma criança emitir a resposta verbal mãe, e não a resposta verbal pai, em uma comunidade verbal cuja língua é o Português. Uma característica comumente atribuída a essa categoria de operante verbal é que a resposta refere-se a ou descreve diretamente o seu evento controlador. O mando, diferentemente de todas as outras categorias, teria sua análise funcional vinculada a condições de privação ou estimulação aversiva às quais o organismo pode ser exposto, e seu estabelecimento dependeria de um reforçador específico. Por exemplo, uma pessoa com febre, em uma sala de aula com ar condicionado ligado, poderia pedir ao professor que desligue o ar. Nesse caso, dada uma estimulação aversiva presente (a variável motivacional, i.e. o evento que altera o valor de certo reforçador), a resposta verbal apontaria um reforçador específico.

Portanto, parece ser que na busca por variáveis de controle ambientais, Skinner (1957) acaba categorizando modalidades de respostas verbais principalmente como função de sua relação com a modalidade do estímulo controlador, deixando em segundo plano o processo de aquisição dos operantes verbais, enquanto produtos de suas consequiências. Por exemplo, o comportamento textual e o de cópia referentes a uma mesma palavra escrita, apresentariam os mesmo antecedentes e o mesmo tipo de consequiência seria responsável pela sua emissão, mas por apresentarem produtos de respostas de modalidades (elemento formal) diferentes (a palavra falada ou a palavra escrita pelo organismo que se comporta), seriam categorias diferentes e, portanto, a função psicológica também diferiria.

\section{III) Responder relacional e operantes verbais de segunda ordem}

Outros tipos de operantes verbais podem ser controlados por eventos ambientais cujas propriedades de controle não podem ser determinadas em termos das características formais desses eventos. Trata-se aqui do responder verbal do tipo relacional que implica a aprendizagem de estruturas gramaticais, a produção de novas estruturas que demonstram a recombinação de componentes, o tatear ações etc. Se, por um lado, Skinner (1957) atrelou sua categorização dos operantes verbais elementares a propriedades físicas de estímulos antecedentes como controladoras das respostas verbais, por outro, exemplos de operantes verbais dos tipos "se", "então", "portanto", "desta forma", "talvez", ou adjetivos como "familiar" (usado no artigo de Stemmer, 1990), "amável", ou verbos como "receber", "segurar", "manter", não apresentam descrição do controle de estímulo específico responsável, pelo menos não no texto de Skinner (1957).

Ao lidar com algumas respostas verbais desse tipo, Skinner (1957) lança mão do termo autoclítico - comportamentos de segunda ordem que descrevem, qualificam ou comentam respostas verbais primárias e, portanto, alteram o efeito da primeira resposta sobre o ouvinte, no sentido de tornar o discurso organizado e efetivo de acordo com as circunstâncias vigentes.

Skinner (1957) (ver também Ribes, 1999; Moore, 2000 e Passos, 2004) apresentou quatro tipos de operantes autoclíticos:

Autoclíticos Descritivos: por meio dos autoclíticos descritivos, o falante pode descrever seu próprio comportamento verbal, algo que ele disse ou que dirá, além de poder descrever a força de suas respostas, as variáveis que as controlam, assim como algo referente a suas condições emocionais ou motivacionais. Exemplos seriam iniciar sentenças com frases dos seguintes tipos: Eu tenho certeza, Eu penso que, Possivelmente, Eu afirmei etc;

Autoclíticos Qualificadores: qualificam um tacto de tal forma que a intensidade ou a direção da conduta do ouvinte em relação ao tacto elementar é modificada. Entre este tipo de autoclítico estão a afirmação e a negação, como também alguns advérbios (certamente, 
unicamente, muito etc) e sufixos (oso - medroso, jeitoso etc). Por exemplo, uma afirmação do tipo "Sim!", pode persuadir o ouvinte a aceitar determinada descrição de eventos como tactos relativamente puros, enquanto o uso de advérbios ou sufixos, em afirmações do tipo: "O garoto é muito inteligente" ou "O garoto e medroso", pode modificar o comportamento do ouvinte em relação à fala (e até mesmo ao garoto) em função da qualificação adicional apresentada pelo falante. De certa forma, variações deste tipo de autoclítico funcionam como mandos sobre o ouvinte;

Autoclíticos Quantificadores: indicam ou uma propriedade da conduta do falante ou as circunstâncias responsáveis por tal propriedade. Neste tipo estão inclusos os artigos de número e gênero (o, a, os, as, um, uns, uma, umas) - sendo estes os exemplos mais destacados -, e os adjetivos e advérbios de quantidade ou tempo (poucos, muitos, todos, alguns, sempre, talvez). É interessante indicar aqui que a definição gramatical de advérbio é semelhante à definição dada por Skinner a essa categoria. Por exemplo, Paschoalin e Spadoto (1996) definem que "Advérbio é a palavra que indica as circunstâncias em que ocorre a ação verbal" (p. 117);

Autoclíticos Relacionais: este tipo de autoclítico seria controlado por relações entre operantes verbais básicos. Exemplos seriam as preposições, conjunções, inflexões da predicação, pontuação, concordância temporal, de gênero e número e a própria ordenação sintática das palavras. A função fundamental deste tipo de autoclítico seria a de permitir a organização do comportamento verbal em unidades maiores do que aquelas possibilitadas pelos operantes verbais elementares, estabelecendo relações internas entre operantes verbais distintos.

Desde modo, seguindo a categorização skinneriana, os componentes da sentença "A blusa é amarela" poderiam ser analisados da seguinte forma: a partícula $A$ seria um autoclítico quantificador, que indicaria que o comentário refere-se a um objeto particular (blusa). As palavras "blusa" e "amarela" seriam tactos elementares, e o verbo "é" (uma inflexão do verbo ser) seria um autoclítico relacional, que especifica uma propriedade do objeto do qual se está falando.

Analisando o tratamento de Skinner do operante verbal autoclítico, Ribes (1999) afirma que os autoclíticos são identificados a partir de três tipos de componentes verbais: 1) partículas gramaticais (abreviações, terminações, pontuação); 2) Unidades gramaticais especiais (artigos, preposições, conjunções, pronomes, adjetivos e advérbios) e 3) Frases e orações que têm efeitos de referência ou predicação sobre outras frases e orações. Partindo da classificação destas unidades dentro da categoria autoclítica, pode-se observar que existe uma dificuldade em se identificar as unidades autoclíticas independentemente de sua morfologia e funções gramaticais.

Finalizando, Skinner (1957) não descreve adequadamente o controle de estímulos neste tipo de repertório verbal, afirmando somente que sua função é diferente da referencial, até agora comentada. Partindo da morfologia e função gramaticais para a classificação do autoclítico, Skinner afasta-se novamente de sua proposta inicial, uma análise funcional do discurso do falante.

\section{IV) Operantes verbais relacionais: complementações à abordagem skinneriana}

\section{Nathan Stemmer e a noção de Eventos Ostensivos}

Para Stemmer (1992) a aprendizagem do comportamento de falante teria como condição necessária a aprendizagem prévia do comportamento de ouvinte. Esta última seria possível em função da exposição do indivíduo, dentro de sua comunidade verbal, a eventos ostensivos. Estes eventos se caracterizariam pela exposição do indivíduo ao pareamento de um estímulo verbal a um estímulo não-verbal, sendo o primeiro uma referência ao segundo. Como um exemplo simples, um pai poderia dizer a seu filho ao apontar para uma boneca: "Isto é uma boneca", ou, colocando o mesmo estímulo não-verbal em uma outra categoria, poderia dizer: "Isto é um brinquedo". Em ambos os casos, haveria o pareamento, em um dado contexto verbal, de um estímulo verbal a um estímulo nãoverbal. Dada a exposição a $n$ exemplares deste tipo de evento ostensivo, $\operatorname{com} n>=1$, o sujeito poderia responder apropriadamente a novos estímulos ambientais, idênticos ou similares àqueles aos quais já foi exposto, em função da formação de classes de generalização entre eventos, subjetivas a cada organismo.

Uma vez que o organismo passasse a responder adequadamente como ouvinte, apontando para objetos ou eventos correspondentes no ambiente físico quando verbalmente requerido, ou respondendo verbalmente o nome de um objeto ou evento, sua produção verbal poderia ser diferencialmente reforçada e estabelecida. Seria possível então, a aprendizagem do comportamento de falante.

Da mesma forma, poderia ser estabelecido o operante verbal relacional e sintático/gramatical. No primeiro caso, isso se daria por meio da exposição repetida a eventos ostensivos cujo estímulo não-verbal implicaria uma relação entre eventos já conhecidos 
pelo sujeito. Como um exemplo citado pelo próprio Stemmer (1992):

\begin{abstract}
Suponha que Mary já tenha aprendido as palavras "mamãe" e "a bola", mas nunca tenha ouvido a palavra "segurar" (ou outras variantes do verbo "segurar"). A garota está agora olhando para sua bola (ex., ela emite "Bola?"), e seu pai responde "Mamãe segura a bola”. Ouvir essa pronúncia pode dirigir a atenção da garota aos objetos nomeados por "mamãe" $e$ "a bola" - a mãe e a bola - e conseqüentemente à relação específica a qual os dois objetos pertencem (stand for): a relação de segurar. Se isso dá saliência suficiente para a relação, o evento ostensivo - o pareamento de "mamãe segura a bola" com o evento de segurar - pode tornar a garota um ouvinte competente da estrutura " $x$ segura y”. (p. 75)
\end{abstract}

A aprendizagem e a generalização do comportamento sintático/gramatical para Stemmer (1990) se explicaria, também, via exposição a eventos ostensivos que gerariam o controle por propriedades estruturais e generalização deste controle. Uma estrutura deveria conter uma palavra relacional capaz de unir elementos já conhecidos pelo sujeito, sendo a palavra relacional (ou as várias palavras relacionais de diversas estruturas), também já conhecida. A aprendizagem de uma estrutura dependeria da exposição repetida do indivíduo a regularidades estruturais em um contexto verbal que tornasse a relação a ser aprendida mais saliente. Após ter aprendido uma estrutura gramatical na voz ativa "Papai está segurando o livro", a criança poderia ouvir, numa mesma situação de estimulação (estímulo não-verbal), a seguinte sentença passiva: "O livro está sendo segurado pelo papai". A mesma situação de estimulação seria agora capaz de gerar os dois tipos de sentenças, explicando desta forma a produtividade gramatical.

Em ambos os casos, de aprendizagem de uma palavra relacional ou de aprendizagem de estruturas, Stemmer $(1990,1992)$ abraça a proposta skinneriana de comportamento verbal em termos dos processos básicos de discriminação e generalização adotados por Skinner para explicar o controle do evento verbal. A diferença entre os autores seria que Stemmer apresenta a descrição dos processos de estabelecimento do comportamento de ouvinte como pré-requisito para o comportamento de falante, além da consideração de que respostas verbais relacionais apresentam-se sob controle de propriedades funcionais ou estruturais de eventos ambientais. Os termos propriedades funcionais ou estruturais referem-se à aquisição de controle por propriedades não-fisicas do evento controlador. São propriedades que só podem ser conhecidas quando se conhece a história de condicionamento do indivíduo. O significado de uma palavra tal como casual para um indivíduo, por exemplo, não se encontraria em uma propriedade física comum a todos os eventos que ele chama de casuais.

\section{Murray Sidman e o Paradigma da Equivalência de Estímulos}

Enquanto Skinner (1957) incluiu comportamentos verbais de nomear objetos, eventos, ou utilizar-se de outros símbolos para se referir a fatos do ambiente físico e físico-social na categoria de comportamento verbal primária denominada tacto, Sidman (1994, 2000) sugeriu que a nomeação envolve um outro processo resultante da contingência, a equivalência entre os estímulos que dela participam. A atividade simbólica observada em humanos teria como base um processo de formação de classes de equivalência entre estímulos relacionados arbitrariamente.

A proposição de que classes de equivalência seriam a base do comportamento simbólico, teve sua origem em um estudo realizado por Sidman (1971). O autor, que na época concentrava-se na investigação dos processos subjacentes à compreensão de leitura, observou que um indivíduo com retardo mental profundo que apresentava em seu repertório comportamental algumas relações entre palavras faladas e figuras, além de relações entre as mesmas palavras faladas e as palavras impressas correspondentes, foi capaz de relacionar adequadamente estas palavras impressas às figuras correspondentes, e vice versa, sem o treino direto destas relações. Logo, dado o treino de algumas discriminações condicionais entre estímulos, o autor observou que novas discriminações emergiam sem treino direto.

Discutindo a natureza desses desempenhos emergentes, Sidman e Tailby (1982) propuseram um sistema descritivo cuja finalidade era identificar a formação de classes de estímulos equivalentes no mesmo contexto de treino e teste de discriminações condicionais entre estímulos, que atestaria a substitutabilidade funcional entre estímulos fisicamente diferentes (mas ver Tonneau, 2001a, 2001b para um tratamento crítico deste sistema).

Nesse sistema descritivo, ensinadas as relações condicionais entre pares de estímulos de dois conjuntos, por exemplo A e B, e, também, de mais dois conjuntos, p. ex. B e C, de tal forma que os pares formados pelos estímulos dos conjuntos A e B possuam 
um elemento em comum com os pares formados pelos estímulos dos conjuntos B e C, quais sejam, os estímulos do conjunto $\mathrm{B}$, pode-se dizer que as relações entre os elementos desses conjuntos são de equivalência se, sem ensino adicional, forem demonstradas as seguintes novas relações condicionais: 1) entre cada estímulo de cada conjunto e ele mesmo, que corresponde à propriedade reflexiva; 2) a relação reversa de cada uma das discriminações condicionais ensinadas, invertendo-se funções de modelos e comparações no procedimento de pareamento, ou seja, as relações condicionais $\mathrm{BA}$, simétricas às relações ensinadas $\mathrm{AB}$ (o mesmo sendo válido para as relações $\mathrm{BC}$ e $\mathrm{CB}$ deste exemplo paradigmático); e 3) entre os estímulos A e $C$, possíveis pela mediação do membro comum nas relações ensinadas entre A e B, e B e C, correspondendo à propriedade transitiva. Por fim, 4) entre os estímulos $\mathrm{C}$ e $\mathrm{A}$, correspondendo às relações simétrica e transitiva das relações ensinadas entre os estímulos dos conjuntos A e B, e B e C. Essas últimas relações emergentes comprovariam, de forma direta, a equivalência entre os estímulos que participam das discriminações condicionais ensinadas e, portanto, a formação de classes de estímulos equivalentes a partir dos pares ordenados dos conjuntos A, B e C.

Voltando aos resultados da pesquisa de Sidman (1971), dado que o indivíduo em questão apresentava um repertório verbal precário no início de seu treino e que inúmeros esforços fracassados já haviam sido implementados na tentativa de ensinar habilidades de leitura a esse indivíduo, hipotetizou-se (Sidman, 1994) deste treino e de sua replicação (Sidman \& Cresson, 1973) que os organismos provavelmente são capazes de relacionar arbitrariamente eventos ambientais que foram apresentados juntos em contingências de reforço consistentes. $\mathrm{O}$ comportamento relacional seria um resultado necessário da exposição a contingências de reforçamento, uma vez que os organismos apresentariam uma capacidade comportamental relacional básica, como o é a capacidade de aprender. Desta forma (diferentemente da posição de Hayes, 1994, como será visto a seguir), as relações entre estímulos que comprovariam a substitutabilidade entre eles decorreriam necessariamente da contingência, como um processo comportamental selecionado filogeneticamente, e não seriam decorrentes de um treino direto de quadros relacionais reflexivos, simétricos, transitivos.

O fato para o qual Sidman $(1994,2000)$ chama a atenção é que, embora a linguagem apresente esta característica relacional, no sentido de que palavras (faladas ou impressas) e seus referentes são, muitas vezes, substituíveis no controle do comportamento, a capacidade de responder adequadamente e da mesma forma a estímulos arbitrários que constituem classes talvez se apresente como uma vantagem evolutiva para as diversas espécies. Quando se fala em classes de estímulos arbitrárias, está-se falando em eventos ambientais que não apresentam similaridade física, mas que se apresentaram relacionados em contingências de reforço, e são, portanto, capazes de controlar respostas comuns de um organismo, e cujas funções são passíveis de modificação, gerando novos comportamentos, em decorrência da modificação da função dos elementos aos quais eles se relacionam. A teoria da equivalência seria, dessa forma, um passo em direção à explicação dos processos implicados na aquisição e desenvolvimento de comportamentos simbólicos.

Visto desta forma, os processos envolvidos na aprendizagem de discriminações simples ou na aprendizagem de relações não seriam mais ou menos complexos para um ou outro organismo (humanos ou nãohumanos). O que se torna mais complicado na aprendizagem de relações em organismos não-humanos seria o desenvolvimento de procedimentos experimentais eficientes que dêem conta de programar contingências suficientes para gerar o desempenho planejado (Galvão, Barros, Goulart, Mendonça \& Rocha, 2002). A visão de Sidman (1994) estaria de acordo com uma abordagem evolucionista que defende a continuidade estrutural e funcional entre as espécies, lembrando sempre que não se busca, por meio dela, uma compreensão total da complexidade da linguagem humana.

\section{Steven C. Hayes e a Teoria do Quadro Relacional}

Uma outra extensão da explicação skinneriana do comportamento verbal foi proposta por Hayes (1991, 1994; ver também Hayes, Barnes-Holmes \& Roche, 2001) com a apresentação da Teoria do Quadro Relacional. $\mathrm{O}$ autor e colaboradores recorrem ao conceito de quadros relacionais (relational frame) como características do funcionamento lingüístico. Hayes afirma que o comportamento verbal é responder relacional aplicável arbitrariamente; aplicável arbitrariamente porque as propriedades que controlam esse responder não são características físicas específicas de um evento ambiental. Um exemplo interessante a esse respeito foi apresentado por Mcllvane (2003) em seu artigo comentário sobre a proposta de Hayes: “...a relação de "dominância" pode ser descrita independentemente das características físicas daqueles que dominam e daqueles que são dominados" (p. 30). O dominante ou o dominado não apresentam características físicas específicas que os coloquem em uma ou 
outra categoria; a relação entre eles é que estabelece o tipo de responder que se dá frente a cada um.

Para Hayes e cols. (2001) o responder relacional aplicável arbitrariamente, estabelecido por meio da exposição a diversos exemplares de um tipo de relação (p. ex. A é maior que B) apresenta três apresenta três características fundamentais: 1) Interdependência mútua (mutual entailment): quando o sujeito aprende uma relação entre A e B, a relação reversa também é estabelecida. Além do exemplo da relação simétrica (na qual a relação entre os elementos é a mesma), se um indivíduo é capaz de responder à relação $\mathrm{A}>\mathrm{B}$, ele deve ser também capaz de responder à relação $\mathrm{B}<\mathrm{A}$; 2) Interdependência combinatorial (combinatorial entailment): seriam relações derivadas entre estímulos em função da relação destes com membros comuns. A transitividade seria um exemplo, mas aqui novamente, outras relações poderiam apresentar tal característica: se uma pessoa é capaz de responder às relações $A>B$ e $\mathrm{B}>\mathrm{C}$, ela deve ser capaz de responder à relação $\mathrm{A}>\mathrm{C}$ e 3) Transformação de função: a modificação da função de um estímulo que participa de uma relação geraria necessariamente a transformação da função de outros estímulos que se relacionem àquele (ver exemplo a seguir).

De uma maneira mais específica, para Hayes e cols. (2001) a exposição a inúmeros exemplares de treino seria necessária para a aprendizagem do responder relacional que, uma vez "estruturado", resultaria em uma classe de respostas generalizada, que, como tal, deveria ser definida somente em termos de suas conseqüências. Os processos subjacentes a esta aprendizagem seriam os mesmos de qualquer operante. $\mathrm{O}$ autor está propondo que operantes de um tipo diferente resultam do treino lingüístico. $\mathrm{O}$ responder relacional não implicaria a existência de um novo processo subjacente a sua aprendizagem, seria apenas um resultado diferente dos processos já conhecidos - um tipo de operante até então não descrito pela abordagem analítico-comportamental, mas que se fundamentaria em processos já descritos pela mesma.

Os quadros relacionais aprendidos iriam além do estabelecimento de relações de equivalência entre estímulos. Hayes (1994) não está falando somente da formação de classes arbitrárias entre estímulos. Hayes está falando também de relações entre eventos ambientais que não necessariamente resultam em classes, mas que, por permanecerem em uma relação, a modificação da função de um estímulo deve modificar, necessariamente, a função de outro estímulo àquele relacionado. $\mathrm{O}$ autor não fala apenas em transferência de funções, característica dos estímulos que participam de classes de equivalência; ele fala em transfor- mação de funções. Exemplificando, dada uma relação de oposição entre $\mathrm{A}$ e $\mathrm{B}$, se $\mathrm{A}$ adquire a função de estímulo aversivo, B apresentará a função neutra ou, opostamente, de reforçador positivo.

No caso da formação de classes de equivalência, o controle de estímulos emergente derivado da aprendizagem de discriminações condicionais entre conjuntos de estímulos (um tipo de responder relacional) observado no desempenho de sujeitos humanos em laboratório decorreria, para Hayes, do fato de que diversos exemplares de relações simétricas e transitivas já haviam sido ensinados durante a exposição desse indivíduo a sua comunidade lingüística.

O responder relacional seria, portanto, comportamento aprendido e deveria, como tal, 1) desenvolverse no decorrer da aprendizagem dos organismos; 2) apresentar variação (vários tipos de relações poderiam ser aprendidos); 3) permanecer sob controle de estímulos (que o autor chama de dica contextual) e 4) ser estabelecido e mantido por reforçamento (para o autor, o reforçamento inicial seria arbitrário e social).

Assim, a formação de quadros relacionais (e o responder relacional aplicável arbitrariamente) tornaria possível a emergência de novos desempenhos verbais que se adequariam aos quadros relacionais já estabelecidos pelo treino, quando uma dica contextual adequada estivesse presente.

Um problema na proposta de Hayes $(1991,1994)$ refere-se ao fato de que as pesquisas realizadas pelo autor e colaboradores não fornecem a descrição do processo de aquisição inicial do quadro relacional. Hayes (1994) cita um estudo longitudinal com um bebê como participante, no qual os autores observaram que aos 19 meses o responder relacional de tipo simétrico surgia como função de determinado treino relacional. $\mathrm{O}$ estudo citado é incompleto por não apresentar o treino necessário para o estabelecimento do quadro aprendido, apresentando apenas o momento em que determinado tipo de responder relacional arbitrário surgiu na história da criança. $\mathrm{O}$ mesmo pode ser dito sobre seus estudos com participantes universitários. Os resultados dessas pesquisas nada dizem sobre o processo de aquisição desse tipo de operante, uma vez que a história pré-experimental desses indivíduos deve, muito provavelmente, tê-los munido com um repertório relacional bem estabelecido.

Concluindo, a teoria de Hayes acrescenta novos conceitos (ainda que problemáticos) para o entendimento do comportamento verbal (mais especificamente relacionados aos seus aspectos semânticos), mas, de forma diferente de Stemmer, não expande o conhecimento da área sobre as possíveis variáveis de controle do comportamento gramatical ou sintático e suas extensões. 


\section{CONSIDERAÇÕES FINAIS}

\section{0 problema de verificação de controle de estímulos}

Ao relatar sobre o controle ambiental do comportamento verbal em seu livro, Skinner (1957) comenta: "A propriedade (do evento ambiental) correlacionada com reforçamento deve ser especificada em termos físicos, se queremos permanecer dentro da estrutura de uma ciência empírica" (Skinner, 1957, p. 117). Assim foi feito durante sua classificação. Após o estabelecimento de controle de estímulos via reforçamento direto, para explicar a emergência de novos comportamentos, Skinner utiliza o processo de generalização. Desta forma, o comportar-se adequadamente frente a novos eventos ambientais foi explicado em termos de generalizações de propriedades físicas de estímulos que já haviam adquirido controle sobre o comportamento.

Existem dados claros de que quando o organismo é exposto a contingências de reforço com o estabelecimento de controle de estímulos, ele passa a responder também a estímulos fisicamente similares àqueles aos quais ele foi diretamente exposto durante sua aprendizagem $^{5}$. É concebível dentro dos princípios da análise do operante verbal de Skinner que, uma vez aprendido o tacto "cadeira", nós sejamos também capazes de emitir este mesmo operante verbal frente a estímulos similares, sendo que esta habilidade pode se iniciar de forma imprecisa, tornando-se cada vez mais precisa em função da modelagem fornecida pela comunidade verbal por meio da aplicação de reforço, punição, extinção.

A abstração seria outro processo capaz de descrever comportamento emergente, também em termos da generalização; neste caso, uma ou mais propriedades de um evento que já adquiriu controle sobre uma resposta do organismo, passaria a controlar respostas a outros eventos que, embora dissimilares, apresentem alguns aspectos em comum com o primeiro. $\mathrm{O}$ mesmo poderia ocorrer com o comportamento textual ou ecóico em suas possíveis unidades mínimas - a recombinação silábica baseada em propriedades físicas dos estímulos positivos, relativa ao controle de unidades silábicas sobre o responder. Para as categorias verbais elementares, Skinner (1957) apresentou exemplos de operantes verbais controlados por eventos físicos relativamente passíveis de descrição.

Esta posição defendida por Skinner para explicar aqueles processos (ex. generalização) indica um tipo de referencialismo, no sentido de localizar o controle da resposta em propriedades físico-químicas dos eventos ambientais. O problema de verificar quais são os requisitos para o comportamento emergente e para a aprendizagem relacional depara-se, de início, com uma dificuldade em se avaliar o próprio estabelecimento de controle de estímulos no treino direto dos operantes verbais; ou seja, de sabermos quais aspectos ou propriedades adquirem o controle do comportamento, como ponto de partida para a generalização ou qualquer outro processo responsável pela emergência de novos comportamentos. A pergunta inicial deveria ser: Quais propriedades de um evento podem adquirir, de fato, o controle sobre determinada resposta? Como propriedades tão específicas (de cada um dos referentes das diferentes categorias verbais elementares) podem resultar de um treino na comunidade verbal que dependa de reforçadores generalizados? E a consequiência necessária destas perguntas é: Quais processos seriam responsáveis pela formação de classes de estímulos ou de classes de respostas generalizadas necessários à emergência de novos comportamentos a partir das respostas originalmente aprendidas?

$\mathrm{O}$ fato relevante aqui é admitir que a análise do comportamento encontra bastante dificuldade até mesmo para definir o controle de estímulos em um problema de discriminação aparentemente muito simples. A teoria da coerência de topografia de controle de estímulos (McIlvane, Serna, Dube \& Stromer, 2000) aplicada à análise da aprendizagem de discriminações simples e discriminações condicionais por primatas não-humanos (Barros, Galvão \& Mcllvane, 2002) indica que, mesmo em procedimentos cujo evento ambiental planejado para adquirir o controle sobre o comportamento é uma simples figura ou relações entre figuras bidimensionais, os sujeitos podem, em grande parte dos casos, responder sob controle de propriedades destes eventos as quais não correspondem às especificadas pelos experimentadores. Além disso, existe uma imensa dificuldade em se verificar experimentalmente quais propriedades adquiriram, de fato, o controle da resposta (Serna \& Carlin, 2001). Este é o problema da indeterminação das propriedades definidoras dos conceitos, já reconhecido há muito tempo por diversos filósofos analíticos (p. ex. Quine, 1960; Wittgenstein, 1953)

\section{Classes de estímulos, relações entre estímulos ou classes de resposta generalizadas?}

Considerando-se exemplares de objetos e a nomeação destes, dentro da abordagem sidmaniana, uma relação de equivalência deveria se estabelecer entre duas classes de generalização - a de um objeto determinado e a de seu nome correspondente (que pode ser a palavra falada pelo indivíduo, por exemplo). No 
caso do indivíduo letrado, diversas classes de generalização estariam ainda envolvidas nestas classes de estímulos equivalentes: a palavra ouvida pelo indivíduo (que deveria responder de forma adequada a tal estímulo quando requerido), a palavra lida pelo indivíduo, a escrita etc, o mesmo ocorrendo sobre todas as habilidades envolvidas no uso atual do instrumento lingüístico pelo homem.

Um outro problema surge: as palavras faladas poderiam ser consideradas estímulos de uma classe, ou respostas de uma classe generalizada. Seriam do primeiro tipo para Sidman no procedimento de pareamento ao modelo quando a palavra é falada ao sujeito; seriam do segundo tipo para Skinner quando se trata de um tacto estabelecido. Do que exatamente se está falando? De classes de estímulos ou classes de respostas? Parece haver uma necessidade recíproca de uma para a outra. Só se pode avaliar a formação de classes de estímulos via responder. Mas o responder na maioria das vezes não fornece o controle de estímulos estabelecido.

De forma semelhante, um problema na proposta de Hayes (1994) é certa imprecisão/vagueza no uso dos conceitos que sustentam a sua teoria. Por exemplo, ora ele fala em estímulos relacionados, ora em responder relacional aplicável arbitrariamente. Não está claro se ele está se referindo às relações entre estímulos ou a uma classe de respostas generalizada relativa a cada quadro relacional aprendido (ver Burgos, 2003). Embora cite que o responder relacional seja controlado por dicas contextuais, o contexto não pode ser identificado com controle de estímulos estabelecido sobre o comportamento, paralelo que o autor tenta fazer entre o responder relacional e o operante. Para este autor, uma classe de respostas como o tacto "formiga", por exemplo, também envolveria a aprendizagem de quadros relacionais. Como seria o estabelecimento dessa relação? A exposição a quais tipos de exemplares relacionais seria necessária? Seria exatamente o mesmo tipo de treino do tacto descrito por Skinner (1957)?

Deste modo, ainda que as teorias complementares apresentadas aqui representem certos avanços ao tratamento skinneriano do comportamento verbal (principalmente do tipo relacional), não existe ainda um consenso sobre como exatamente diferentes mecanismos e processos bio-comportamentais atuam na aquisição e no desenvolvimento do comportamento simbólico (Mclvane, 2003; Tomasello, 2003). Conforme a análise realizada neste trabalho, parece ser que um passo importante para o avanço nesta discussão na área da análise do comportamento deveria passar por uma padronização conceitual no que se refere às noções de aprendizagem relacional, classes de estímulos e classes de respostas generalizadas. Uma maior precisão conceitual deve se refletir no estabelecimento de hipóteses claras sobre mecanismos e processos relevantes na aquisição e o desenvolvimento de repertórios verbais, hipóteses estas que devem ser mantidas (e implementadas) ou rejeitadas com bases nos resultados experimentais derivados delas.

\section{REFERÊNCIAS}

Barros, R. S., Galvão, O. F. \& Mcllvane, W. J. (2002). Generalized identity matching to sample in Cebus apella. The Psychological Record, 52, 441-460.

Burgos, J. E. (2003). Laudable goals, interesting experiments, unintelligible theorizing: a critical review of Relational Frame Theory. Behavior and Philosophy, 31, 19-45.

Chomsky, N. (1959). Review of B. F. Skinner's. Verbal Behavior Language, 35, 26-58.

Galvão, O. F., Barros, R. S., Goulart, P. R. K., Mendonça, M. B. $\&$ Rocha, A. C. (2002). Escola de primatas. Estudos de psicologia (Natal), 7, 361-370.

Fodor, J. (1983). The modularity of mind. Cambridge, MA: MIT Press.

Hayes, S. C. (1991). A relational frame theory of stimulus equivalence. Em L. J. Hayes \& P. N. Chase (Orgs.), Dialogues on verbal behavior (pp. 19-40). Reno: Context Press.

Hayes, S. C. (1994). Relational frame theory: a functional approach to verbal events. Em S. C. Hayes, L. J. Hayes, M. Sato \& K. Ono (Orgs.), Behavior analysis of language and cognition (pp. 9-30). Reno: Context Press.

Hayes, S. C., Barnes-Holmes, D. \& Roche, B. (2001). Relational frame theory: a post-skinnerian account of human language and cognition. NY: Kluwer Academic/Plenum.

McIlvane, W. J. (2003). A stimulus in need of a response: a review of relational frame theory: a post-skinnerian account of human language and cognition. The Analysis of Verbal Behavior, 19, 29-37.

McIlvane, W. J., Serna, R. W., Dube, W. V. \& Stromer, R. (2000). Stimulus control topography coherence and stimulus equivalence: reconciling test outcomes with theory. Em J. Leslie \& D. Blackman (Orgs.), Experimental and applied analysis of human behavior (pp. 85-110). Reno: Context Press.

Moore, J. (2000). Words are not things. The Analysis of Verbal Behavior, 17, 143-160.

Paschoalin, M. A. \& Spadoto, N. T. (1996). Gramática: teoria e exercícios. São Paulo: FTD.

Passos, M. L. (2004). Bloomfield e Skinner: língua e comportamento verbal. Rio de Janeiro: NAU Editora.

Pinker, S. (1994). The language instinct. New York: William Morrow.

Peterson, N. (1978). An introduction to verbal behavior. Grand Rapids, MI: Behavior Associates.

Quine, W. V. O. (1960). Word and object. Cambridge, MA: Harvard University Press.

Ribeiro, A.F. (2004). O que é o comportamento verbal. Em C. E. Costa, J. C. Luzia \& H. H. N. Sant'Anna (Orgs.), Primeiros 
passos em análise do comportamento e cognição, Vol. 2 (p. 67-76). Santo André, SP: Esetec.

Ribes, E. (1999). Teoría del condicionamiento y lenguaje: Un análisis histórico y conceptual._Guadalajara: Taurus.

Serna. R. W. \& Carlin, M. T. (2001). Guiding visual attention in individuals with mental retardation. Em L. M. Glidden (Org.), International review of research in mental retardation, Vol. 24. (pp. 321-357). San Diego, CA: Academic Press.

Sidman, M. (1971). Reading and auditory-visual equivalence. Journal of Speech and Hearing Research, 14, 5-13.

Sidman, M. (1994). Equivalence relations and behavior: a research story. Boston: Authors Cooperative.

Sidman, M. (2000). Equivalence relations and the reinforcement contingency. Journal of the Experimental Analysis of Behavior, 74, 127-146.

Sidman, M. \& Tailby, W. (1982). Conditional discrimination vs. matching to sample: an expansion of the testing paradigm. Journal of the Experimental Analysis of Behavior, 37, 5-22.

Sidman, M. \& Cresson, O. (1973). Reading and crossmodal transfer of stimulus equivalences in severe retardation. American Journal of Mental Deficiency, 77, 515-523.

Skinner, B. F. (1957). Verbal behavior. New Jersey: Prentice Hall.
Stemmer, N. (1990). Skinner's verbal behavior, Chomsky review, and mentalism. Journal of the Experimental Analysis of Behavior, 54, 307-315.

Stemmer, N. (1992). The behavior of the listener, generic extensions, and the communicative adequacy of verbal behavior. The Analysis of Verbal Behavior, 10, 69-80.

Tomasello, M. (2003). Constructing a language: A used-based theory of language acquisition. Cambrigde, MA: Harvard University Press.

Tonneau, F. (2001a). Equivalence relations: a critical analysis. European Journal of Behavior Analysis, 2, 1-33.

Tonneau, F. (2001b). Equivalence relations: a reply. European Journal of Behavior Analysis, 2, 99-128.

Wittgenstein, L. (1953). Philosophical investigations. New York: Macmillan.

Recebido: 10/09/2005

Revisado: 16/11/2005

Aceito: 15/12/2005

\section{Notas:}

${ }^{1}$ Ver Peterson (1978) e Ribeiro (2004) para apresentações mais detalhadas dos operantes verbais básicos.

${ }^{2}$ A similaridade formal (SF) seria uma relação entre um estímulo que evoca uma resposta e o produto daquela resposta. Para que haja similaridade formal nesta relação o estímulo e o produto da resposta devem, necessariamente: 1) ser ambos da mesma modalidade; 2) Assemelharem-se quanto aos padrões físicos ou seqüenciais. (Um exemplo seria o responder ecóico, cujo estímulo controlador seria vocal e o produto da resposta controlada, também vocal. A palavra falada mesa, controlaria a resposta mesa, cujo produto seria um som com padrões físicos e seqüenciais semelhantes aos do estímulo controlador) (Peterson, 1978).

${ }^{3}$ A correspondência ponto-a-ponto seria a relação entre um estímulo verbal e a resposta que ele controla, cujos aspectos definidores são os seguintes: 1) O estímulo verbal e a resposta devem ser compostos de dois ou mais componentes; 2) O primeiro componente do estímulo deve controlar o primeiro componente da resposta, assim para os demais componentes, não importando se o estímulo e a resposta pertencem à mesma modalidade. (Peterson, 1978).

${ }^{4}$ Um exemplo seria a resposta verbal Luiz Inácio Lula da Silva dado o estímulo verbal antecedente Presidente do Brasil. Variações deste tipo de operante verbal são diariamente estabelecidas no repertório verbal de crianças por meio dos procedimentos tradicionais de ensino das escolas brasileiras e de outros países.

${ }^{5}$ Ver Journal of the Experimental Analysis of Behavior, 2002, Vol. 78 (3), edição especial "Categorization and Concept Learning”.

\section{Sobre os autores:}

Ana Leda de Faria Brino: Mestre em Teoria e Pesquisa do Comportamento - Universidade Federal do Pará; Doutoranda do Programa de Pós-graduação em Teoria e Pesquisa do Comportamento - UFPA. Bolsista de doutorado do CNPq (abrino@gmail.com)

Carlos B. A. de Souza: Doutor em Ciencias de la Conducta - Centro de Estudios e Investigaciones en Comportamento - Universidad de Guadalajara - México - Programa de Pós-graduação em Teoria e Pesquisa do Comportamento - UFPA. Pesquisador 2 do CNPq (carlos.souza@pesquisador.cnpq.br) 